\title{
CAUSATIVIZACIÓN PERIFRÁSTICA EN TOBA DEL ESTE Y DEL OESTE DE LA PROVINCIA De Formosa (Argentina)
}

\author{
Periphrastic causativization in Toba from Eastern and Western Formosa \\ PROVINCE (ARgENTINA)
}

\author{
Causativité périphrastiQue de la langue toba dans l'est et l'ouest \\ de LA PRovince de Formosa (Argentine)
}

\section{Raúl Eduardo González \\ Licenciado y doctor en Letras, Universidad Nacional del Nordeste (Argentina). Becario posdoctoral, Consejo Nacional de Investigaciones Científicas y Técnicas (CONICET), Instituto de Investigaciones Geohistóricas (IIGHI)-CONICET. Profesor adjunto regular, cátedra "Culturas originarias en el Gran Chaco", Facultad de Artes, Diseño y Ciencias de la Cultura (FADYCC), Universidad Nacional del Nordeste (UNNE). \\ Mailing address: La Rioja 145 5A, 3500 Resistencia (Chaco), Argentina E-mail: raul_eduardogonzalez@ yahoo.com.ar}

\section{María Belén Carpio}

Doctora en Letras por la Universidad Nacional de Córdoba (Argentina), magíster en Lingüística por la Universidad de Sonora (México), licenciada en Antropología por la Universidad Nacional de Rosario (Argentina). Investigadora asistente del CONICET, Instituto de Investigaciones Geohistóricas (IIGHI)-CONICET. Profesora adjunta de la cátedra "Lenguas indígenas y de inmigración habladas en el NEA" de la FADYCC, Instituto de Investigaciones Geohistóricas (IIGHI)-CONICET- UNNE.

Mailing address: La Rioja 145 5A, 3500 Resistencia (Chaco), Argentina. E-mail: belenvenado@yahoo.com.ar

\section{RESUMEN}

El objetivo de este trabajo es analizar, desde la perspectiva funcionalista, las construcciones causativas perifrásticas en toba del este y del oeste de Formosa, y comparativamente entre ambas variedades. En especial, se considera el grado de fusión de los predicados de causa y de efecto, el orden y el grado de variabilidad paradigmática de los mismos. La estrategia de causativización perifrástica registrada en toba del este de Formosa no fue descripta en las variedades de Chaco - con las que es mutuamente inteligible - y se demuestra que presenta diferencias, sobre todo en términos de gramaticalización, con la construcción causativa perifrástica del toba del oeste de Formosa. El corpus está compuesto por relatos orales y cláusulas elicitadas, registrados en trabajos de campo realizados en tres comunidades de la provincia de Formosa: Vaca Perdida, al oeste, y barrio Nam Qom y Bartolomé de las Casas, en el este y centro-este. Este artículo constituye una contribución a investigaciones de tipo comparativo de los mecanismos de causativización perifrástica en dos variedades de toba. A su vez, ante la ausencia de registros diacrónicos, la comparación en la sincronía de lenguas genéticamente emparentadas, pero habladas por pueblos que han atravesado procesos etnohistóricos diferenciales, proporciona evidencia empírica que puede permitir postular, a modo de hipótesis, procesos de gramaticalización en curso.

Palabras clave: causativos sintácticos, gramaticalización, lingüística tipológicofuncional, variabilidad paradigmática verbal, guaycurú, toba

\begin{abstract}
The aim of this paper is to analyze, from a functionalist perspective, periphrastic causative constructions in Toba from Eastern and Western Formosa province, and comparatively between the two varieties. Particularly, this paper focuses on the features of degree of fusion of the cause and effect predicate, their order, and paradigmatic variability. The periphrastic causative constructions in Toba from Eastern Formosa that were described were previously undetected in the mutually intelligible varieties in the province of Chaco. Besides, findings show that this construction differs, especially in terms of its degree of grammaticalization, from periphrastic construction in Toba from Western Formosa. The corpus is
\end{abstract}

Received: 2016-11-24 / Accepted: 2017-06-05

DOI: $10.17533 /$ udea.ikala.v22n03a05 
composed of oral texts and elicited clauses collected during fieldwork pursued in three communities: Vaca Perdida in Western Formosa, and Nam Qom neighborhood and Bartolomé de las Casas in Eastern and Middle-Eastern Formosa. This paper is a contribution to comparative studies on periphrastic causative constructions of two varieties of Toba. In the absence of diachronic sources, the synchronic comparison of genetically related languages, but spoken by ethnohistorically different peoples, provides empirical evidence to hypothetically postulate grammaticalization processes in course.

Keywords: syntactic causatives, grammaticalization, functional typological linguistics, verbal paradigmatic variability, Guaycuruan, Toba

\section{RÉSUMÉ}

L'objectif de ce travail est d'analyser les constructions causatives périphrastiques du toba dans l'est et l'ouest de Formosa depuis les perspectives fonctionnelle et comparative. Plus précisément, nous considérerons le degré de fusion, tout comme l'ordre et le degré de variabilité paradigmatique des prédicats de cause et d'effet. La stratégie de causativité périphrastique observée dans le toba de l'est de Formosa n'avait pas été décrite, à ce jour, dans les variétés du Chaco -avec lesquelles il est pourtant mutuellement intelligible. Nous démontrerons que cette variante de l'est de Formosa présente des différences avec celle de l'ouest, surtout dans le plan de la de grammaticalisation. Le corpus est composé par des récits oraux et des clauses colligés durant le travail de terrain effectué dans trois communautés de la dite province, soit: Vaca Perdida dans l'ouest ainsi que Nam Qom et Bartolomé de las Casas, dans l'est et le centreest. Cet article constitue une contribution aux recherches de type comparatif des mécanismes de causativité périphrastique dans deux variétés du toba. Toutefois, devant l'absence de registres diachroniques, la comparaison dans la synchronie de langues génétiquement apparentées, mais parlées par des peuples qui ont traversé des processus ethno-historiques différentiels fournit l'évidence empirique qui peut permettre de postuler, en guise d'hypothèse, des processus de grammaticalisation en cours.

Mots-clés : causatifs syntaxiques, grammaticalisation, linguistique fonctionnelle typologique, variabilité paradigmatique des verbes, guaycurú, toba 


\section{Introducción}

El toba es una lengua que pertenece a la familia guaycurú, junto al pilagá, mocoví, caduveo y las ya extintas abipón y mbayá (Tovar y Larrucea de Tovar, 1984, p. 43). La causativización ha sido analizada, con diversos grados de profundidad, en lenguas de la familia: mocoví (Gualdieri, 1998, pp. 256-266; Carrió,2012),pilagá(Vidal,2001,pp.166-171,360364), toba de la provincia de Chaco (Buckwalter, [1980] 2001, pp. 488-493; Klein, 1981, p. 56; Censabella, 2008, y Porta y Messineo, 2010), toba del oeste de Formosa (Carpio, 2012, pp. 139147, 2015), toba del este de Formosa (González, 2015, pp. 121-126), abipón (Najlis, 1966, pp. 3536) y caduveo (Sándalo, 1995, pp. 154-172). El objetivo de este trabajo es profundizar el estudio de la causativización en toba del este y del oeste de Formosa, en especial en lo que respecta a las construcciones perifrásticas. Se analiza en cada variedad y comparativamente, desde la perspectiva funcionalista, el grado de fusión de los predicados de causa y de efecto, el orden y el grado de variabilidad paradigmática de los mismos (posibilidad de conmutar los morfemas de persona y número), atentos a posibles rutas de gramaticalización.

En la región del Gran Chaco argentino, los tobas habitan principalmente en las provincias de Chaco, Formosay, en menor proporción, en el este de Salta. Como resultado de procesos migratorios internos, existen en la actualidad importantes enclaves en la ciudad de Rosario (provincia de Santa Fe) y en el conurbano de la ciudad de Buenos Aires. Según los datos del último censo (Instituto Nacional de Estadística y Censos -INDEC-, 2012), la población estimada a nivel nacional de personas que se identifican como tobas es de 126.967. En la provincia de Formosa se ubican principalmente en el sureste (departamento Pilcomayo, Formosa capital y departamento Laishí), en el centro (departamentos Pilagá, Pirané y Patiño) — donde habitan mayoritariamente grupos pilagá-, y en el noroeste (departamentos de Matacos y Bermejo).
Las principales diferencias etnohistóricas entre los tobas orientales y occidentales formoseños radican en los grupos indígenas con los que entablaron relaciones de alianza o de guerra, y en la naturaleza del contacto con la población criolla. Los tobas del este fueron enemigos de grupos maká que habitaban la franja derecha del río Pilcomayo, en el Chaco paraguayo, y también se consideraban enemigos de sus vecinos pilagás. Los tobas del oeste, por el contrario, fueron aliados de los pilagás y establecieron matrimonios interétnicos e intercambios económicos de diverso tipo, además de relacionarse con otros grupos tobas hacia el norte de su territorio, en lo que actualmente es Bolivia. Por su parte, los tobas occidentales, junto a los pilagás, se enfrentaban a los chulupíes o nivaclés, que ocupaban la franja izquierda del Pilcomayo, río arriba de sus territorios.

Además, los territorios orientales de la provincia de Formosa fueron objeto de exploración e intentos de ocupación por parte de los españoles ya desde los siglos XVII y XVIII, por medio de diversas estrategias: ocupación militar, fundación de pequeñas ciudades agrícolas y establecimiento de misiones jesuíticas. El oeste de la provincia, en cambio, permaneció durante mucho tiempo inexplorado y fuera de los circuitos económicos hasta mediados del siglo Xx (Mendoza y Wright, 1989, p. 245).

Ambos grupos hablan variedades lingüísticas ininteligibles entre sí, mientras que los tobas del este declaran que pueden entenderse con los hablantes de la provincia de Chaco y los del oeste con los pilagá.

Las variedades de toba del este y del oeste de Formosa comparten características generales, como tendencia a la polisíntesis, oposición verbo/nombre y marcación de núcleo en lo que respecta a la relación entre predicado y argumentos. No poseen marcación de caso ni adposiciones. En cuanto al orden de los constituyentes, las combinaciones más 
utilizadas son: VS ${ }^{1}$ y PVA (cuando P se expresa como pronombre libre). Los índices pronominales en los verbos presentan alineación escindida, de acuerdo con la jerarquía de persona: predominantemente nominativo-acusativo cuando se trata de los participantes en el acto de habla y tripartito en la tercera persona. Poseen una construcción pasiva no promocional que es utilizada mayoritariamente sobre verbos transitivos, pero también, aunque en menor medida, sobre verbos intransitivos, mediante la cual se destopicaliza el agente de la cláusula activa, aunque sin promoción del argumento P. La pasiva no promocional se expresa con morfología verbal concomitante, por medio del prefijo qa- $\sim$ qo- $\sim$ qodi-. Estas variedades cuentan, además, con un variado número de afijos verbales que codifican locación y dirección. Algunos de ellos se comportan como aplicativos. Los verbos carecen de expresión morfológica de tiempo. Solamente la categoría de aspecto es codificada por medio de afijos verbales (Carpio, 2012;

\section{Marco teórico-metodológico}

En lo que respecta a la relación entre los mecanismos formales y los rasgos semánticos de la causativización, Givón (2001, p. 75) plantea que en las lenguas que poseen construcciones causativas perifrásticas y morfológicas, las primeras tenderán a ser utilizadas para expresar causación más débil, es decir, cuando el causado - manipulado, en términos del autor- es agentivo humano, mientras que las segundas tenderán a expresar situaciones causativas más fuertes que implican un causado no agentivo, inanimado. Por su parte, Shibatani y Pardeshi (2002, p. 86) analizan los distintos mecanismos formales de codificación de las situaciones causativas, en especial en lo que respecta a la productividad de los mismos o el grado de transparencia morfológica del elemento

$1 S$ = argumento único de la cláusula intransitiva; $A=$ argumento más parecido al agente de cláusula transitiva; $\mathrm{P}=$ argumento más parecido al paciente de cláusula transitiva (Comrie, 1978, p. 331).

causativo. De este modo, Shibatani (2002, pp. 4, 6) sostiene que la estrategia de codificación de la causativización será más explícita cuanto más difícil sea llevar a cabo el evento causado. La dificultad de ejecución del evento causado se relaciona con la configuración espacio-temporal de la situación causativa según los tipos de eventos involucrados y los rasgos semánticos que caracterizan al causante y al causado. De este modo, cuanto más se aproxima la situación causativa al polo de la causación directa, mayor será la probabilidad de que se seleccionen estrategias de causativización menos transparentes morfológicamente (causativos léxicos y formas morfológicas irregulares) frente a estrategias más transparentes morfológicamente o más productivas (morfología regular y construcciones perifrásticas).

Comrie (1989, p. 171) plantea dos rasgos formales centrales para el análisis translingüístico de las construcciones causativas: 1) la codificación gramatical del causado, y 2) el grado de fusión formal de los predicados de causa y efecto en un único predicado. Ambos rasgos son analizados por Song (1996) desde una perspectiva que sitúa las construcciones causativas junto al resto de las construcciones morfosintácticas de la lengua, especialmente en lo que respecta al control de la densidad de frases nominales por cláusula, al grado de fusión en la codificación de causa y efecto, y al carácter implicativo o no de la relación entre los eventos que componen la situación causativa. En este sentido, Song (1996, pp. 20-68; 2013) distingue las construcciones causativas compactas de las analíticas de tipo secuencial (o AND) y de propósito (o PURP). Considera como compactas las construcciones léxicas, morfológicas y también aquellas en las que la causa es codificada por un ítem léxico que constituye una unidad gramatical con el verbo de efecto. A su vez, este autor plantea que en las construcciones analiticas de tipo secuencial ocurren dos cláusulas que contienen el predicado de causa y el de efecto, y el orden de las mismas refleja icónicamente la prioridad lógica y temporal de la causa sobre el efecto. Es la secuencia temporal no 
reversible de los eventos lo que caracteriza a este tipo de construcciones más que la presencia del coordinante. En cambio, en las construcciones analíticas de propósito, la relación entre el evento causante y el evento causado es no implicativa. Translingüísticamente, no es extraño que la causación indirecta o no implicativa sea codificada mediante un verbo de "decir", puesto que la manipulación humana puede ser tanto verbal como no verbal (Maldonado y Nava, 2002; Song, 1996; Givón, 2001, entre otros).

La comparación de las estrategias de causativización perifrástica en toba del este y del oeste de Formosa es analizada en términos de un continuum de gramaticalización. La gramaticalización es entendida como el proceso que permite que determinada forma o construcción de tipo léxica asuma una función gramatical, o bien un morfema o construcción asuma una función aún más gramatical.

En la gramaticalización, las unidades sintácticas atraviesan distintos momentos: 1) pierden autonomía sintáctica, 2) debilitan su significado referencial, 3) suelen erosionarse o debilitarse fónicamente y perder peso fonológico, 4) debilitan o pierden la distribución sintáctica contextual originaria, y 5) suelen cambiar su estatus gramatical e integrarse a nuevos paradigmas, entre otros. $\mathrm{Al}$ mismo tiempo, las formas ganan en cohesión gramatical con otros morfemas y la capacidad de ocurrir en nuevos contextos más generales, alejados de los etimológicos originarios.

En un doble y simultáneo proceso de pérdida y ganancia, el cambio es visto como acumulativo, de manera que los nuevos valores más abstractos y más gramaticales pueden coexistir con las formas y los significados léxicos de base (Heine y Kuteva, 2002, p. 2; Company Company, 2009, pp. 71-72). Como plantea Lehmann (1985, pp. 307-308), la pérdida de autonomía de un signo lingüístico o el aumento de su grado de gramaticalización puede ser evaluado, paradigmática y sintagmáticamente, en tres aspectos: 1) peso: integridad y extensión de la construcción en la que interviene; 2) cohesión: grado de integración en un paradigma y de unión con otros signos dentro del sintagma, y 3 ) variabilidad: posibilidad de utilizar otros signos en su lugar o de omitirlo y de desplazarse en una construcción.

El trabajo de campo fue realizado en tres comunidades de la provincia de Formosa: Vaca Perdida (departamento de Bermejo), al oeste, y barrio Nam Qom y la excolonia secular Bartolomé de las Casas, en el este y centro-este. En todos los casos se trabajó con hablantes bilingües toba-castellano, que poseen la lengua indígena como primera lengua. Los textos fueron traducidos con consultante de referencia y segmentados morfema a morfema. Estos relatos orales (historias de vida, relatos históricos, comentarios, etc.) fueron obtenidos a pedido de los investigadores, o bien inducidos a partir de un estímulo audiovisual. Además, se contó con cláusulas elicitadas con distintos consultantes de referencia.

En cuanto al análisis del corpus, la estrategia metodológica de base consiste en la conmutación de morfemas y cláusulas, lo que nos permite reconocer y clasificar los morfemas y agruparlos en paradigmas, así como establecer sus reglas de combinación en la cadena sintagmática. La comparación de estructuras oracionales se realizó en cada lengua y entre las dos lenguas analizadas.

\section{Resultados de la investigación}

Se describieron las estrategias de causativización en toba del oeste y del este de Formosa, con especial énfasis en las construcciones perifrásticas, y luego se compararon en función del grado de fusión de los verbos de causa y de efecto, el orden y la variabilidad paradigmática de los mismos. A su vez, se propone, a modo de hipótesis, un continuum de gramaticalización de las construcciones causativas. 


\section{Causativización en toba del oeste de Formosa}

En toba del oeste de Formosa fueron descriptas distintas estrategias morfosintácticas de causativización (Carpio, 2016, 2015, 2012), las cuales pueden ser sistematizadas en un continuum de síntesis compuesto por formas compactas y construcciones perifrásticas. Las estrategias compactas de causativización implican: 1) la alternancia de índices pronominales tipo I y II, ${ }^{2}$ o entre índices pronominales tipo I, si se trata de verbos de base que codifican locación y dirección; 2) el uso del sufijo - $a G a-t$ 'modificador de valencia no pacientivo-instrumental' (también se registró, aunque con menor frecuencia, el uso del sufijo - $t$ 'instrumental' solo como causativizador) cuando los verbos de base son intransitivos de estado, logro y realización (véase ejemplo 1), ${ }^{3}$ o junto al sufijo - $n$ 'agentivo', cuando se trata de verbos de base intransitivos de actividad (véase ejemplo 2); los sufijos $-a G a-n-a G a-n$ 'modificador de valencia no pacientivo-agentivo-modificador de valencia no pacientivo-agentivo' son utilizados cuando los verbos de base son transitivos ${ }^{4}$ (véase ejemplo 3). La estrategia perifrástica de causativización está compuesta por el verbo de causa en ('hacer'), en el que se codifica persona y número del causante, al que se pospone el verbo de efecto en el que se expresan persona y número del causado; y si es transitivo, número del paciente. Esta estrategia fue detectada en todos los verbos del corpus analizado, independientemente de la transitividad, el aspecto léxico inherente de los mismos o el tipo de índice pronominal (4)-(8). Los verbos de traslación solo admiten la estrategia de causativización perifrástica (8).

(1) a. w-akák ha? ñ-ahóm l-ó?o

$3 \mathrm{I}^{5}$-quebrarse F.DEM 1POS.ALIE-puerta 3POS.INAL-dueño

'Se quebró mi llave'.

b. ho? nogotolé-k y-akag-aGá-t

DEM niña-MASC 3I-quebrarse-MV-INST

l-ó?o

3POS.INAL-dueño

'El niño quebró mi llave'.

(2) a. ní?-me noGotolé-k w-anók

DEM-ENDOF niña-MASC 3I-mamar

'El niño mama'.

b. ha-ñi?-me qa? y-anog-aGá-n ñi? qóGot

F-DEM-ENDOF ADV 3I-mamar-MV-AGT DEM hijo

'Ella amamantó al hijo'.

2 En toba del oeste de Formosa fueron analizados tres tipos de índices pronominales que codifican al argumento único de una cláusula intransitiva $(S)$ y al argumento más parecido al agente $(\mathrm{A})$, cuya selección está pautada desde el lexicón de la lengua (Carpio, 2012, pp. 108-131).

3 En ambas lenguas, ejemplificamos solo las construcciones causativas que son específicamente relevantes en términos del continuum de causativización propuesto.

4 Los verbos de base semánticamente transitivos, pero sintácticamente intransitivos agentivos do 'he ('habla'), 'dohe ('cocina'), de 'loki ('pelea') y di? 'yaqo ('pesca') son causativizados a través de los sufijos -aGa-n-aGa-n. En este contexto morfosintáctico, esta serie de sufijos confluye en el aumento de la transitividad de la cláusula de base, habilitando la codificación del causado, del mismo modo que cuando estos sufijos disminuyen y aumentan la transitividad en bases verbales semántica y sintácticamente transitivas.

5 Convenciones: 1, 2, 3 = primera segunda y tercera persona; $3 \mathrm{IPOS}=$ poseedor de tercera persona indeterminado; $\mathrm{AB}=$ direccional tipo I 'hacia abajo'; $\mathrm{AD}=$ direccional tipo II 'adentro de'; $\mathrm{ADV}=$ adverbio; AGT = agentivo; $\mathrm{AL}=$ aplicativo 'alativo'; $\mathrm{ALIE}=$ alienable; $\mathrm{BEN}=$ benefactivo; $\mathrm{COMP}=$ complementizador; 
(3) a. ha-dá?-me yawó y-iyó há?-me n-ekemáGaho F-DEM-ENDOF mujer 3I-lavar DEM.F-ENDOF 3POS.ALIE-pantalón

'La mujer lavó su pantalón'.

b. ha-dá?-me yawó d-iyóGo-n

F-DEM-ENDOF mujer 3I-lavar.MV-AGT

'La mujer lava'.

c. y-iyóGo-n-aGa-n ha-dá?-me yawó

3I-lavar.MV-AGT-MV-AGT F-DEM-ENDOF mujer

'Hizo que la mujer lave [por ejemplo, si contrató a una mujer para que lave]'.

(4) $\boldsymbol{h}$-en $\boldsymbol{w}$-a' $\boldsymbol{k a k} \quad \tilde{k} i$ ?

1I-hacer 3I-quebrarse DEM 1POS.ALIE-puerta 3POS.INAL-dueño

'Hice quebrar la llave de mi puerta'.

(5) Ø-en h-oyín há?-me hoganqaté

3I-hacer 3II-llorar F.DEM-ENDOF vacuna

'Le hizo llorar la vacuna'.

(6) Ø-en y-óte hén-me nogóp

3I-hacer 1III-orinar DEM-ENDOF agua

'Me hizo orinar el agua'.

(7) qo-y-en h-oñip-iñi

PAS-3I-hacer 1I-sentarse-AB

'Hacen que me siente'.

(8) Ø-en t-áyngi hén-me nogóp

3I-hacer 3I-AB.AD DEM-ENDOF agua

'Hizo que [alguien] entre al agua'.

En las construcciones causativas perifrásticas, cuando el verbo de base es transitivo, los argumentos codificados como frases nominales son: el causante - que puede ser omitido- y el último

COORD = coordinante DEM = determinante demostrativo; ENDOF = endofórico; EXIST.NEG = existencial negativo; $\mathrm{F}=$ femenino; $\mathrm{GR}=$ grupo restringido; $\mathrm{I}$, II, III = índice pronominal tipo I, II y III; INAL = inalienable; INST = instrumental; INT = direccional 'hacia adentro'; MASC $=$ masculino $;$ MV $=$ modificador de valencia no pacientivo; $\mathrm{NEG}=$ negación $; \mathrm{NOM}_{5}=$ nominalizador deverbativo 'nombre de acción voluntaria'; NPROG = no progresivo; $\mathrm{PAC}=$ paciente PAS $=$ pasiva no promocional; $\mathrm{PDEM}=$ pronombre demostrativo; $\mathrm{PL}=$ plural; POS = poseedor; $\mathrm{PROG}=$ progresivo $\mathrm{SG}=$ singular; negrita: destaca el morfema sobre el cual se está focalizando el análisis y para lo cual se ilustra con el ejemplo. participante afectado de la cadena causal, el paciente, obligatoriamente expresado luego del complejo verbal causativo (9).

No obstante, es posible identificar al causado por medio dela indexación en el verbo de efecto cuando se trata de un participante en el acto de habla (10).

En cambio, cuando el causado no es un participante en el acto de habla, es necesario recurrir a diversas estrategias morfosintácticas para codificarlo como frase nominal plena no opcional. Si se desea expresar el causado en estas construcciones, es necesario modificar la transitividad de alguno de los verbos que expresan la situación causativa. Es posible antipasivizar el verbo de efecto (11b) o pasivizar el verbo de causa (12b). 
En las construcciones causativas perifrásticas, en toba del oeste de Formosa se observan rasgos de fusión de ambos verbos, puesto que no es posible interrumpir la adyacencia física de los mismos. El verbo de causa siempre precede al de efecto. Además, solo ocurre un marcador aspectual en el verbo de efecto con alcance sobre todo el complejo verbal (13). No se registró el uso de morfología aspectual en el verbo de causa.

Sin embargo, los verbos de causa y de efecto poseen autonomía sintáctica, puesto que reciben flexión de persona y número del argumento causante y causado, respectivamente, y pueden ocurrir junto al prefijo de pasiva no promocional. En el verbo de causa se codifica persona y número del causante, pero permanece invariante respecto del valor de número de los argumentos del verbo de efecto. Este último recibe flexión de persona y número del causado y, si es transitivo, número del paciente. El verbo de causa -en ('hacer') solo recibe flexión de persona y número del causante (14), a diferencia de cuando ocurre como un verbo factitivo simple, donde recibe flexión de persona y número de $\mathrm{A}$ y número de $\mathrm{P}(15)$.

(9)h-en y-ilolé ho? níyaga chitá?

1I-hacer 3I-freír DEM pescado grasa

'Hice que [alguien] fría la grasa de pescado'.

(10) Ø-en h-ikatén ñi? máyo

3I-hacer 3I-mirar DEM pájaro

'Me hace mirar al ave'.

(11) a.Ø-en $\boldsymbol{y}$-ihók ${ }^{6} \quad \tilde{n} i$ ? -me nogotolé-k

3I-hacer 3I-vacunar DEM-ENDOF niña-MASC

'Hizo que [alguien] vacune al niño'.

b.Ø-en d-ehóg-aGa-n ha-ñi?-me qoqt-é

3I-hacer 3I-vacunar-MVNP-AGT F-DEM-ENDOF hijo-F

'Hizo que la hija vacune'.

(12) a.ha-dá?-me qa? Ø-en y-inoGón ha-dá?-me l-edág anqate F-DEM-ENDOF ADV 3I-hacer 3I-quebrar F-DEM-ENDOF 3POS.INAL-lápiz 'Ella hizo que [alguien] quiebre el lápiz'.

b.qá?et ñí-me dario qo-y-ín y-inogón epáq

ayer DEM-ENDOF Darío PAS-3I-hacer 3I-quebrar palo

'Ayer, hicieron que Darío quiebre el palo'.

(13) Ø-en h-alolá-tak hén-me ló?omaga

3I-hacer 1I-toser-PROG DEM-ENDOF frío

'El frío me está haciendo toser'.

(14) a. h-en al-ahóqtayi dá?-me n-aqták 1I-hacer 2III-sostener DEM-ENDOF 3IPOS-palabra 'Hago que sostengas la palabra'.

b. h-en al-ahóqtayi-lo dá<a>?-me n-aqtáq-a

1I-hacer 2III-sostener-PL DEM $<$ PL $>$-ENDOF 3 IPOS-palabra-PL

'Hago que sostengas las palabras'.

6 El verbo yi 'hok también significa 'moler'. 

(15) $\boldsymbol{h}-\boldsymbol{e}<\boldsymbol{p}>\boldsymbol{n} \quad$ dá $<a>$ ?-me l-apyá? $-t e$ 1I-hacer $<$ PL.PAC $>$ DEM $<$ PL $>$-ENDOF 3POS.INAL-zapato-PL 'Hice sus zapatos'.

Esta diferencia en la densidad de codificación de persona y número de los argumentos del verbo -en ('hacer'), dependiendo de si funciona como verbo factitivo simple o verbo de causa en una construcción causativa perifrástica, puede deberse a que, como plantea Cristófaro (2003, p. 250), las situaciones en las que se comparten participantes son particularmente propicias para motivar una menor densidad de codificación en una de las cláusulas que conforman la construcción compleja. En este sentido, dado que, en las construcciones causativas perifrásticas, los argumentos $\mathrm{P}$ del verbo de causa y A/S del verbo de efecto son correferentes y es posible recuperar la información acerca de quién es el causado a través de la codificación de persona y número en el verbo de efecto, el hablante, en consonancia con el "principio de recuperación de información"
(Cristófaro, 2003, p. 249) vinculado a la economía sintagmática, no reitera esos rasgos en el verbo principal.

\section{Causativización en toba del este de Formosa}

En toba del este de Formosa se describieron mecanismos compactos de causativización, cuya selección depende de la transitividad del verbo de base. Cuando los verbos de base son intransitivos, se recurre a la alternancia de los índices pronominales de tercera persona, o a la sufijación de - $a G a$ 'modificador de valencia no pacientivo' junto a $-t$ 'instrumental' (16), y - $n$ 'agentivo' (17) o se utiliza solo este último morfema (18). Cuando los verbos de base son transitivos,

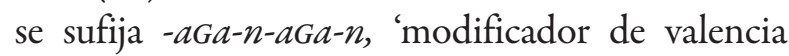
no pacientivo-agentivo-modificador de valencia no pacientivo-agentivo' (19). A su vez, se registró una estrategia de causativización perifrástica (20).

(16) a. $w$-akak

3I-quebrar

'Se quebró'

b. y-akag-aGa-t a-na ?epaq

3I-quebrar-MV-INST F-DEM árbol

'Rompe el árbol'.

(17) a. d-achol-ta

3I-temblar-NPROG

'Tiembla'.

b. ya-chol-aGa-n a-na ?epaq

3I-temblar-MV-AGT F-DEM árbol

'Sacude ese árbol'.

(18) a. ha-damaga d-alemata

F-PDEM 3I-enojarse

'Ella se enoja/está enojada'.

b. ?am y-alemata-n

2SG 3I-enojarse-AGT

'Te hace enojar'.

7 En los ejemplos en toba del este de Formosa no se indica la posición del acento, puesto que es demarcativo, siempre ocurre en posición final absoluta de palabra. 


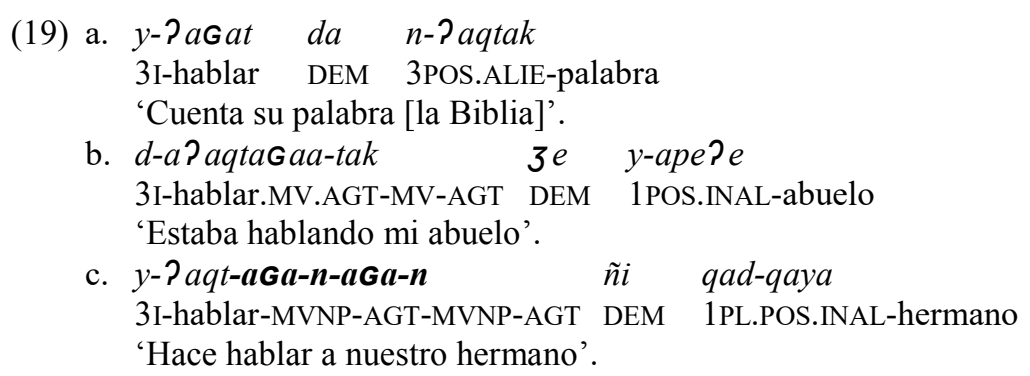

La estrategia de causativización perifrástica registrada en toba del este de Formosa no fue descripta en las variedades de Chaco - con las que es mutuamente inteligible - y presenta diferencias, sobre todo en términos del grado de gramaticalización en lo que respecta a la variabilidad paradigmática de sus constituyentes verbales, con la construcción causativa perifrástica del toba del oeste de Formosa (4)-(14). En toba del este de Formosa, esta construcción está compuesta por el verbo de efecto antipasivizado mediante los sufijos - aGa 'modificador de valencia no pacientivo' y - $n$ '-agentivo', seguido del complementizador $n a$, más el verbo de causa codificado invariantemente como qoyin ('dicen') (20)-(21). No es posible con-jugar esta forma del verbo "decir", sino que se expresa siempre por medio de la tercera persona $y$ - - paradigma defectivo - y el prefijo de pasiva no promocional qo- (variante morfofonológica del prefijo qa- en toba del este de Formosa). El carácter cristalizado de este verbo nos señala que se encuentra en un avanzado proceso de gramaticalización y los hablantes en general lo traducen como "hacer" más que "decir", con lo cual su sentido coercitivo para inducir una acción también se halla altamente convencionalizado.

En (20) podemos apreciar, inmediatamente antepuesta a la construcción causativa analítica, su contraparte morfológica mediante los sufijos -aGa-n-aGa-n. La alternancia de construcciones causativas morfológica y perifrástica a partir de una misma base verbal se ilustra en (22)-(23). La diferencia semántica entre ambas reside en el carácter más directo de la causación en las primeras.

A su vez, en uno de los relatos orales que conforman nuestro corpus en toba del este de Formosa ocurre el verbo nqoyin con la noción de 'ser causante' (24)-(25). Se trata de una forma verbal muy similar a la que observamos en las construcciones causativas perifrásticas. A su vez, Buckwalter (1980 [2001], pp. 148, 259) presenta dos verbos similares: nqoyin y qanqoyin, traducidos como "lo considera" y "lo hacen", respectivamente. El hecho de que la forma nqoyin pueda recibir el prefijo de pasiva no promocional qa-confirma que se trata de un verbo pleno.

(20) qo-y-an-aGa-n-aGa-n, d-an-aGan na qo-y-in qataq PAS-3I-dar-MV-AGT-MV-AGT 3I-dar-MV-AGT COMP PAS-3I-decir COORD 'Le hacen plantar, le dicen que plante, también'.

(21) d-ekes-ogo-n na qo-y-in

3I-cortar-MV-AGT COMP PAS-3I-decir

'Le dicen que corte'.

(22) a. qa-y-asot-aGa-n-aGa-n

PAS-3I-mover los pies-MV-AGT-MV-AGT

'Le hacen patear'.

b. s-asot-aga-n na qo-y-in

1I-mover los pies-MV-AGT COMP PAS-3I-decir

'Me dicen que patee'. 
(23) a. qa-y-?on-aGa-n-aGa-n

PAS-3I-cantar-MV-AGT-MV-AGT

'Le hacen cantar'.

b. d-Pon-aGa-n na qo-y-in

3I-cantar-MV-AGT COMP PAS-3I-decir

'Le dicen que cante'.

(24) qa-y-alawat qayqa na n-qoyin

PAS-3I-matar EXIST.NEG COMP 3II-ser causante

'Matan y no hay quien sea el causante [culpable]'.

(25) hi-ketete-w?a ka Ø-?etek qayqa na n-qoyin

3I-quemar-INT.AL DNP 3I-decir EXIST.NEG COMP ${ }^{8}$ 3II-ser causante

'Quema, prende fuego y dice que no hay culpable'.

De acuerdo con los mecanismos de causativización señalados por Dixon (2000, pp. 33-40), la construcción perifrástica que analizamos en toba del este de Formosa presenta una tendencia que contradice la generalización translingüística, ya que como se observa en (20), (21), (22b) y (23b), el verbo principal de la construcción perifrástica - que puede ocurrir con cualquiera de los índices pronominales en todas las personas, es decir, puede ser conjugado - es el que codifica el evento causado, mientras que el verbo de causa "decir" se encuentra en un nivel de dependencia sintáctica, ya que ocurre pospuesto a la raíz deíctica na, que se utiliza en la lengua como uno de los complementizadores más frecuentes para introducir complementos verbales (26)-(27). El orden verbo de efecto + verbo de causa en toba del este de Formosa puede dar cuenta de un estadio sincrónico intermedio en el proceso de gramaticalización, cuyos polos están representados por verbos factitivos simples y verbos de decir, por un lado, y sufijos causativos, por el otro.

$$
\begin{aligned}
& \text { (26) sa-yanaq-tak [na n-wi?] } \\
& \text { 1PLI-saber.1PLI-PROG COMPL 3I-llegar } \\
& \text { 'Estamos sabiendo que llega'. }
\end{aligned}
$$

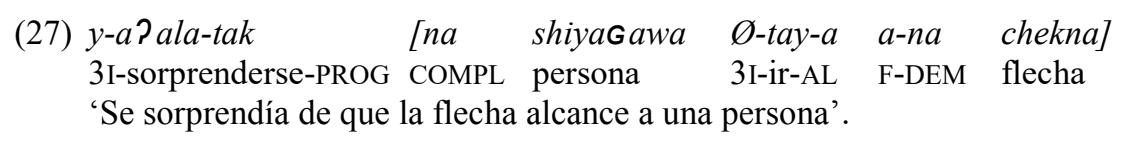

Teniendo en cuenta la similitud morfológica con una de las posibilidades de codificación del verbo -en ('hacer') en toba del oeste de Formosa (7) y el uso del verbo nqoyin ('ser causante') en toba del este de Formosa (24)-(25), el análisis del verbo causativo con valor de 'hacer' no puede descartarse definitivamente. En consecuencia, planteamos que la construcción perifrástica compuesta por: verbo-aGa-n + na + qoyin 'dicen', constituye una estrategia gramaticalizada, en la cual el verbo "decir" ha perdido el valor semántico original para inmovilizarse en tercera persona pasivizada y, progresivamente, adquiere el valor de 'hacer', aunque con un agente de tipo desconocido y humano. La

8 En toba del este de Formosa, los complementizadores que permiten introducir cláusulas de complemento son isomórficos con los determinantes demostrativos na "acercándose" y da "parado" (González, 2015, p. 167). 
afirmación de que la noción semántica original del verbo sea "decir" se confirma, de acuerdo con Shibatani y Pardeshi (2002, p. 124), por la restricción que impone este tipo de verbo, en su primera etapa de gramaticalización, para la selección de causantes específicamente humanos o animados. Además, el verbo invariante de causa qoyin en toba del este de Formosa no solo posee afinidad semántica y fonológica con el verbo factitivo simple -en (28), sino también con el verbo de decir que se utiliza para introducir citas directas -e'nak (29), ambos registrados en toba del oeste de Formosa.

\section{Comparación de las construcciones perifrásticas en ambas variedades de toba}

Las construcciones causativas perifrásticas en las variedades de toba analizadas difieren en la ausencia/presencia de complementizador, el orden de los verbos de causa y efecto, y en la mayor o menor variabilidad paradigmática de los índices pronominales en los verbos en cada una de ellas. Es decir, en toba del oeste de Formosa no interviene un complementizador entre el verbo de causa y el de efecto, y este último ocurre siempre pospuesto al verbo de causa. Además, el verbo de causa recibe flexión de persona y número del causante, y el verbo de efecto recibe flexión de persona y número del causado, y si es transitivo, de número del paciente. En cambio, en toba del este de Formosa ocurre el complementizador na entre el verbo de efecto y el verbo de causa, el cual se pospone al verbo de efecto, que siempre se codifica en su versión antipasiva, y el verbo de causa se encuentra cristalizado, invariante en persona y número, es decir, siempre ocurre junto al prefijo de pasiva no promocional y en tercera persona singular.

En este sentido, al comparar las construcciones causativas perifrásticas en toba del este y del oeste de Formosa, es posible plantear un continuum de gramaticalización, en función del grado de variabilidad paradigmática de los verbos que codifican la situación causativa (véase tabla 1): partiendo, en toba del oeste de Formosa, del uso del verbo -en como verbo factitivo simple (28) y del verbo de decir - e'nak (29) con sus respectivas flexiones de persona y número, pasando por la construcción causativa perifrástica compuesta por el verbo -en como verbo de causa con flexión de persona y número de A (causante) + verbo de efecto (32), hasta llegar a la construcción causativa perifrástica en toba del este de Formosa, en la que el verbo -en ocurre cristalizado como qoyin luego del complementizador na y del verbo de efecto intransitivizado (33). En toba del oeste de Formosa, la antipasivización del verbo de efecto (11b) o la pasivización del verbo de causa (12b) constituyen alternativas de codificación como frase nominal del argumento causado. En cambio, en toba del este de Formosa, la única estrategia de causativización perifrástica registrada requiere la antipasivización del verbo de efecto y la pasivización del verbo de causa. A modo de hipótesis, proponemos que el polo más alto en este continuum de gramaticalización está representado por los sufijos $-n$ 'agentivo' y $-t$ 'instrumental', que codifican causación, junto al morfema - $a G a$ 'modificador de valencia no pacientivo' (34)-(35), y en un pequeño número de verbos por sí mismos (Carpio, 2015, pp. 79-81; Censabella, 2008, p. 107). Esta hipótesis está motivada por el aire de familia fonológico y semántico de estos sufijos con los verbos factitivos simples -en ('hacer') (28) y -?et ('hacer, arreglar') (29), y con los verbos de decir -e'nak y -Petak registrados en toba del oeste de Formosa.

En trabajos previos (Carpio y González, en prensa; González, 2015) se muestra que, en la variedad toba del oriente de Formosa, el verbo "decir" sigue otra ruta de gramaticalización, como evidencial de tipo reportativo a partir de la misma raíz verbal. Esta estrategia también se realiza por medio de una forma fosilizada que recurre a la pasiva no promocional qo-y el índice pronominal de tercera persona $y$-, que señala alta transitividad en la lengua. Por medio de qoyitega, el hablante expresa que la información le ha sido brindada por una fuente de información ajena a él, que no ha sido testigo directo de la acción o evento, como en (36) y (37). 
Tabla 1 Construcciones causativas en variedades de toba: continuum de gramaticalización

\begin{tabular}{|c|c|c|c|}
\hline \multicolumn{2}{|c|}{ Toba del oeste de Formosa } & Toba del este de Formosa & $\begin{array}{l}\text { Variedades } \\
\text { de toba de } \\
\text { Formosa y } \\
\text { Chaco }\end{array}$ \\
\hline $\begin{array}{l}\text {-en como verbo factitivo simple (28) } \\
\text { - e nak como verbo de decir (29) } \\
\text { - Pet como verbo factitivo simple (30) } \\
\text { - Petak como verbo de decir (31) }\end{array}$ & $\begin{array}{l}\text {-en como verbo de causa } \\
+ \text { verbo de efecto ( } 32 \text { ) }\end{array}$ & Verbo de efecto antipasivizado $+n a+$ qoyin $(33)$ & $\begin{array}{l}(-a G a)-n(34) \\
(-a G a)-t(35)\end{array}$ \\
\hline \multicolumn{4}{|c|}{$\longleftrightarrow$} \\
\hline$(-)$ & \multicolumn{2}{|c|}{ (Gramaticalización) } & $(+)$ \\
\hline
\end{tabular}

- Toba del oeste de Formosa:

-en ('hacer') como verbo factitivo simple:

(28) maq l-ewáka l-ópok, qamá? Ø-en l-óGok nomás 3POS.INAL-vaca 3POS.INAL-cuero entonces 3I-hacer 3POS.INAL-chirípa 'Nomás que el cuero de vaca tenían, entonces hacían chirípa'.

enák ('decir') como verbo que permite introducir citas directas:

(29) qamá? $\begin{gathered}\text { Øenák hoqá?li } \\ \text { entonces }\end{gathered}$ 3I-decir en aquel tiempo
nombre propio

n-oyín hoqá?li wáyaga lédagay

3II-llorar hace tiempo zorro negro

'Entonces, decía, en aquel tiempo, WayqálaG adi:- lloró, en aquel tiempo, el zorro negro'.

-? et ('hacer, arreglar') como verbo factitivo simple:

(30) a.y-épet $\quad$ ha-gá?-me
3I-hacer
'Hac-DEM-ENDOF el cerco'.

-? étak ('decir') como verbo que permite introducir citas directas:

(31) wayqálagadi qamá? Ø-Pétakhoqá?li ñoqol-qá n-á?na hiyaGadi-pí nombre propio entonces 3I-decir hace tiempo niño-PL 3II-venir gente.PL-COL “Wayqála adi decía, hace tiempo: - hermanos, por ahí viene la gente'.

-en ('hacer') como verbo de causa:

(32) Ø-en qan-ahóykañi hén-me nepíyaga 3I-hacer 1GRII-transpirar DEM-ENDOF calor 'Nos hace transpirar el calor'.

9 Es una prenda de vestir que consiste en un paño rectangular amplio (en este caso de cuero de vaca), pasado entre las piernas y sujeto a la cintura. 
- Toba del este de Formosa:

Verbo- $a G a-n$ como verbo de efecto + (na 'complementizador' + qoyin 'dicen') como verbo de causa:

(33) $d$-ashiw-aGa-n na qo-y-in

3I-fumar-MV-AGT DEM PAS-3I-decir

'Le dicen que fume'.

- Toba del oeste de Formosa (el uso de estos sufijos también se registró en toba del este de Formosa y Chaco):

Sufijos - $a \mathrm{G} a$ - $t$ como causativizadores:

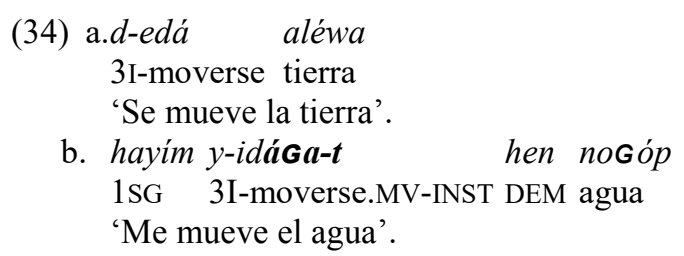

Sufijos - $a \mathrm{G} a-n$ como causativizadores:

(35) a.ñi? nogotolé-k d-eké?e

DEM niña-MASC 3I-comer

'El niño come'.

b. ha-ñi? l-at?é y-iki?y-aGa-n ñi? qóGot

F-DEM 3POS.INAL-madre 3I-comer-MV-AGT DEM niño

'La mamá alimenta al niño'.

(36) $i$-shet da s-?aqt-aGan-em de?eda

3I-poder DEM 1I-contar-MV-AGT-BEN PDEM

'Puede que yo le cuento [a él] sobre eso,

qo-y-i-teg-a ll-iqy-aGak

PAS-3I-decir-PROG-AL 3POS.INAL-camino-NOM

que dicen el caminar'.

(37) na ? ana qo-y-i-teg-a qa-y-ache-tak grabasyon

PDEM PAS-3I-decir-PROG-AL PAS-3I-llevar-PROG grabación

'Esto que dicen están llevando, la grabación'.

De esta manera, ambos procesos de gramaticalización ejemplifican dos de las rutas más frecuentes para verbos como "decir": causación indirecta y evidencialidad reportativa.

\section{Conclusiones}

En este trabajo analizamos las construcciones causativas en toba del oeste y este de Formosa; especialmente, describimos y comparamos la estrategia perifrástica. Demostramos que dicha estrategia difiere en las variedades de toba analizadas en la ausencia/presencia de complementizador, el orden de los verbos de causa y de efecto, y en el grado de variabilidad paradigmática de los índices pronominales en los verbos en cada una de ellas. En toba del oeste de Formosa no ocurre un complementizador entre el verbo de causa y el verbo de efecto. Este último ocurre siempre pospuesto al verbo de causa. Excepto en lo que respecta a indexación de número del argumento $\mathrm{P}$ en el verbo de causa, los verbos que componen la construcción causativa perifrástica presentan la misma variabilidad paradigmática que cuando funcionan como verbos simples. En cambio, en toba del este de Formosa ocurre el 
complementizador na entre el verbo de efecto y el verbo de causa; el orden de estos verbos revierte la prioridad lógica de la causa sobre el efecto, puesto que el verbo de efecto precede al de causa y la variabilidad paradigmática de los constituyentes verbales se ve reducida, en tanto que el verbo de efecto siempre se codifica en su versión antipasiva y el verbo de causa se expresa invariantemente junto al prefijo de pasiva no promocional qoy en tercera persona singular. Fundamentalmente, en función del grado de variabilidad paradigmática de los verbos que ocurren en las construcciones causativas perifrásticas analizadas es posible plantear un continuum en el cual la estrategia perifrástica de causativización en toba del este de Formosa se encuentra más gramaticalizada que en toba del oeste de Formosa.

El presente estudio constituye una contribución a investigaciones de tipo comparativo de los mecanismos de causativización perifrástica en dos variedades de toba. A su vez, ante la ausencia de registros diacrónicos, la comparación en la sincronía de lenguas genéticamente emparentadas, pero habladas por pueblos que han atravesado procesos etnohistóricos diferenciales, proporciona evidencia empírica que puede permitir postular, a modo de hipótesis, procesos de gramaticalización en curso.

\section{Referencias}

Buckwalter, A. ([1980] 2001). Vocabulario toba -Seguido de algunos Apuntes sobre la gramática del idioma toba. Roque Sáenz Peña, Chaco (Argentina): edición del autor.

Carpio, M. B. (2012). Fonología y morfosintaxis de la lengua hablada por grupos tobas en el oeste de Formosa (Argentina). Múnich: Lincom Europa.

Carpio, M. B. (2015). Estrategias de causativización en toba del oeste de Formosa (Flia. guaycurú, Argentina). En Z. Estrada Fernández, A. Fernández Garay y A. Álvarez González (Eds.), Estudios de lenguas amerindias 3. Escenarios de diversidad lingüistica (pp. 73-96). Hermosillo: UniSon.

Carpio, M. B. (2016). Causativización de verbos locativos en toba del oeste de Formosa (guaycurú, Argentina). Lingüistica. Asociación de Lingüistica y Filología de América Latina (ALFAL), 32(2), 47-62.
Carpio, M. B., y González, R. (En prensa). Evidencialidad y modalidad epistémica en dos variedades de toba habladas en Formosa (Argentina). Lexis, XLI. Lima: Pontificia Universidad Católica del Perú.

Carrió, C. (2012). Causación en mocoví. Ponencia presentada en el III Simpósio de linguística ameríndia da Associação de Linguística e Filología da América Latina (ALFAL), Universidad Federal de Río de Janeiro y el Museo Nacional, Río de Janeiro (Brasil).

Censabella, M. (2008). Derivación causativa en lengua toba. En Z. Estrada Fernández, S. Wichman, C. Chamoreau y A. Álvarez González (Eds.), Studies in Voice and Transitivity (pp. 105-125). Múnich: Lincom Europa.

Company Company, C. (2009). Parámetros de gramaticalización en los indefinidos compuestos en el español. En F. Sánchez Miret (Ed.), Romanistica sin complejos. Homenaje a Carmen Pensado (pp. 71-104). Berna: Peter Lang.

Comrie, B. (1978). Ergativity. En W. P. Lehmann (Ed.), Syntactic Typology (pp. 329-394). Sussex: Harvester Press.

Comrie, B. (1989). Language Universals and Linguistic Typology. Syntax and Morphology. Chicago: University of Chicago Press.

Cristófaro, S. (2003). Subordination. Oxford: Oxford University Press.

Dixon, R. M .W. (2000). A typology of causatives: Form, syntax and meaning. En R.M.W. Dixon y A. Aikhenvald (Eds.), Changing Valency. Case Studies in transitivity (pp. 30-83). Reino Unido: Cambridge University Press.

Givón, T. (2001). Syntax. A Functional-Typological Introduction (vol. II). Ámsterdam/Filadelfia: John Benjamins.

González, R. E. (2015). Estudio fonológico y morfosintáctico de la lengua toba hablada en el este de la provincia de Formosa (Argentina). Múnich: Lincom.

Gualdieri, B. (1998). Mocovi (Guaicuru). Fonologia $e$ morfossintaxe (Tesis de doctorado no publicada). Universidade Estadual Campinas, Campinas, Brasil.

Heine, B., y T. Kuteva. (2002). World Lexicon of Grammaticalization. Cambridge: Cambridge University Press.

Instituto Nacional de Estadística y Censos (INDEC) (2012). Censo nacional de población, hogares y viviendas. Censo del Bicentenario. Resultados definitivos, Serie B N. 2 . Buenos Aires, octubre. 
Klein, H. E. M. (1981). Una gramática de la lengua toba: morfologia verbal y nominal. Montevideo: Dirección General de Extensión Universitaria. Ms.

Lehmann, C. (1985). Grammaticalization: Synchronic variation and diachronic change. Lingua e Stile, (20), 303-318.

Maldonado, R., y Nava, F. (2002). Tarascan causatives and event complexity. En M. Shibatani y P. Pardeshi (Eds.), The Grammar of Causation and Interpersonal Manipulation (pp. 157-196). Ámsterdam/ Filadelfía: John Benjamins.

Mendoza, M., y Wright, P. (1989). Sociocultural and economic elements of the adaptation systems of the Argentine Toba: The Nacilamolek and Taksek cases of Formosa Province. En S. Shennan (Ed.), Archaeological Approaches to Cultural Identity (pp. 242-257). Londres: Unwin Hyman.

Najlis, E. L. (1966). Lengua abipona (Archivo de Lenguas Precolombinas, N.o 1). 2 vols. Buenos Aires: Universidad de Buenos Aires, Facultad de Filosofía y Letras, Centro de Estudios Lingüísticos.

Porta, A., y Messineo, M. C. (2010). Capítulo 132: Descripción de la morfología verbal del toba utilizando lenguajes lineales libres de contexto. En V. Castel y L. Cubo de Severino (Eds.), La renovación de la palabra en el bicentenario de la Argentina. Los colores de la mirada lingüistica (pp. 1065-1071). Mendoza: Editorial FFyL, UNCuyo.

Sándalo, F. (1995). A Grammar of Kadiwéu (Tesis de doctorado no publicada). University of Pittsburgh, Pittsburgh, Estados Unidos.

Shibatani, M. (2002). Introduction: Some basic issues in the grammar of causation. En: M. Shibatani (Ed.), The Grammar of Causation and Interpersonal Manipulation (pp. 1-22). Ámsterdam/ Filadelfia: John Benjamins Publishing Company.

Shibatani, M., y Pardeshi, P. (2002). The causative continuum. En M. Shibatani (Ed.), The Grammar of Causation and Interpersonal Manipulation (pp. 85126). Ámsterdam/Filadelfia: John Benjamins.

Song, J. J. (1996). Causatives and Causation. A Universal-Typological Perspective. Londres/Nueva York: Longman.

Song, J. J. (2013). Periphrastic causative constructions. En M. Dryer y M. Haspelmath (Eds.), The World Atlas of Language Structures Online. Leipzig: Max Planck Institute for Evolutionary Anthropology. Recuperado de http://wals.info/chapter/110.

Tovar, A., y Larrucea de Tovar, C. (1984). Catálogo de las lenguas de América del Sur. Madrid: Gredos.

Vidal, A. (2001). Pilagá grammar (Guaykuruan Family, Argentina) (Tesis de doctorado no publicada). University of Oregon, Oregon, Estados Unidos.

How to reference this article: González, R. E., y Carpio, M. B. (2017). Causativización perifrástica en toba del este y del oeste de la provincia de Formosa (Argentina). Íkala, Revista de Lenguaje y Cultura, 22(3), 439-454. DOI: 10.17533/udea.ikala.v22n03a05 